\title{
Shone's Complex and Aortic Dissection: Case Report and Review of a Rare, Underdiagnosed Congenital Heart Disease
}

\section{Steven Sinfield ( $\square$ ssinfiel@hs.uci.edu )}

University of California Irvine https://orcid.org/0000-0003-2820-9132

\section{Sachini Ranasinghe}

University of California Irvine

\section{Stephani Wang}

University of California Irvine

\section{Fernando Mendoza}

Long Beach Memorial Medical Center: Long Beach Medical Center

Ali Khoynezhad

Long Beach Memorial Medical Center: Long Beach Medical Center

\section{Case report}

Keywords: Shone's syndrome, shone's complex, congenital heart disease, aortic dissection, Bentall procedure, aortic aneurysm, LVOT obstruction, patient prosthesis mismatch, MAZE procedure

Posted Date: September 17th, 2021

DOl: https://doi.org/10.21203/rs.3.rs-827168/v1

License: (c) (i) This work is licensed under a Creative Commons Attribution 4.0 International License. Read Full License 


\section{Shone's Complex and Aortic Dissection: Case Report and Review of a Rare, Underdiagnosed Congenital Heart Disease}

\section{Authors}

Steven Sinfield $\mathrm{MD}^{1}$, Sachini Ranasinghe $\mathrm{MD}^{1}$, Stephani Wang $\mathrm{MD}^{2}$, Fernando Mendoza $\mathrm{MD}^{3}$, Ali Khoynezhad MD, PhD ${ }^{3}$

1. Department of Medicine, University of California, Irvine

2. Department of Medicine, Division of Cardiology, University of California, Irvine

3. Memorial Care Heart and Vascular Institute, MemorialCare Long Beach Medical Center

\section{Corresponding Author}

Steven Sinfield, MD

Address for Correspondence:

333 The City Blvd. West, Suite 400

Orange, CA, USA 92868

Tel: $562-822-6761$

Fax: 718-305-1749

Email: ssinfiel@hs.uci.edu 


\section{Abstract}

\section{BackGround}

Shone's complex is a rare congenital heart disease consisting of a variety of left ventricular inflow and outflow tract lesions. Patients typically present in childhood and require early surgical intervention. However, with improved surgical techniques these patients are surviving later into adulthood which comes with a new set of medical complications providers need to be aware of.

\section{Case Presentation}

We present a case of a patient with a complex cardiac history including an incomplete Shone's complex diagnosed in childhood with multiple surgical interventions who presents with persistent symptomatic atrial flutter and a type A aortic dissection in the setting of severe patient prosthesis mismatch. The patient was being followed outpatient for an enlarging chronic aortic aneurysm and was non-compliant with his medications when he presented with sharp chest pain radiating to his back. He was found to have an acute type A aortic dissection and was taken emergently to the operating room for a skirted Bentall procedure, aortic valve replacement, and right sided MAZE.

\section{Conclusions}

Shone's complex is a rare congenital heart disease associated with significant morbidities in adulthood, such as atrial flutter, patient prosthesis mismatch, and aortic dissection. As patients continue to live longer into adulthood with this disease, it is important to raise awareness of this rare syndrome for providers and highlight its potential complications. Further research is needed to determine appropriate guidelines for when to intervene on aortopathy-associated CHD.

\section{Keywords}

Shone's syndrome, shone's complex, congenital heart disease, aortic dissection, Bentall procedure, aortic aneurysm, LVOT obstruction, patient prosthesis mismatch, MAZE procedure 


\section{Background}

Shone's complex (SC) or syndrome is a rare congenital heart disease (CHD) that consists of 4 main anomalies: coarctation of the aorta (CoA), subaortic stenosis, parachute mitral valve (PMV) and supravalvular mitral ring. Complete SC consists of all 4 abnormalities and incomplete SC consists of 2-3 anomalies, which is much more common (1). Most case series now define SC as a combination of mitral valve disease plus at least one level of left heart obstruction. Typical mitral stenosis is most common, with some case series showing it be $70 \%-90 \%$ of all SC cases $(2,3)$.

The pathogenesis of SC is hypothesized to be initially caused by a disruption to the left ventricular inflow tract (LVIT) from a congenital MV anomaly during embryogenesis, leading to underdevelopment of the left ventricle (LV) cavity $(3,4)$. Although previously a disease of unknown cause, a recent study found that $11 \%$ of SC cases were associated with MYH6 de novo mutations, which encodes for the cardiac alpha-myosin heavy chain that is highly expressed in the embryonic heart (5).

Patients typically present in childhood with a spectrum of symptoms including poor feeding, failure to thrive, and signs of reduced cardiac output (6). Management involves cardiac surgery during childhood and most common interventions include surgical coarctation repair, subaortic resection, and mitral valve repair (2). The majority of published studies on SC comes from the pediatric literature. However, with improved surgical techniques within the last 40 years, there has been a $24 \%$ increase in adults living with $\mathrm{CHD}(7)$. As patients with $\mathrm{CHD}$ continue to live longer, this will come with a new set of medical complications in adulthood that providers need to be aware of.

We present a case of a young adult patient diagnosed with incomplete SC during childhood who presented with persistent symptomatic atrial flutter and an acute type A dissection in the setting of a growing proximal aortic aneurysm. This case will highlight a rare form of $\mathrm{CHD}$ and the potential complications of SC management in adult patients.

\section{Case Presentation}

A 27 year old male with a past medical history significant for incomplete SC and persistent atrial flutter initially presented to an outside hospital with $10 / 10$ intensity, sharp chest pain radiating to his back after having a bowel movement. CTA chest at presentation demonstrated an acute type A aortic dissection (AD) (Fig. 1) and patient was transferred to our institution for higher level of care.

He has a complex congenital cardiac history of a shone complex variant including coarctation of the aorta (CoA), subaortic stenosis, pulmonary stenosis, atrial septal defect (ASD), ventricular septal defect (VSD), and mitral stenosis. During his first days of life, he underwent a pulmonary valvotomy, LV 
apical to descending aortic conduit, and ASD/VSD closure. At 1 year of life, he underwent a Konno procedure with a $17 \mathrm{~mm}$ mechanical St Jude aortic valve replacement and mechanical $23 \mathrm{~mm}$ St Jude mitral valve replacement. However, both of his valves were now consistent with severe patient prosthesis mismatch as was now a full-grown adult.

Over the last few years the patient has been having annual monitoring for an enlarging aortic aneurysm, initially noted to be $5.3 \mathrm{~cm}$ in 2016. Despite extensive counseling from his primary care provider and cardiologist, the patient continued to be noncompliant with his beta blocker and participated in heavy weightlifting that placed increased stress on his aortic wall. He continued to have yearly scans and on most recent CTA in 2020, just one month prior to his AD presentation, it demonstrated growth of the thoracic aortic aneurysm to $5.8 \mathrm{~cm}$ with no evidence of dissection or hematoma (Fig 2). He was scheduled for follow up with his congenital cardiologist to discuss surgical options.

On presentation, the patient was alert and oriented with no motor/sensory deficits. He was transferred to the CCU and scheduled for emergent redo sternotomy. In the operating room, the re-do sternotomy was performed through the same skin incision. There were severe adhesions, which lead to nearly 2 hours of dissection and an unintended diaphragmatic RV injury requiring a definitive pericardial patch for repair. The patient was noted to have a large aneurysmal aortic root from the ascending aorta up to the innominate aorta (Fig 3). The ascending aorta was completely resected and hemiarch replacement was performed using a $32 \mathrm{~mm}$ branched vessel Gelweave Ante-Flo graft (copyright Terunmo, Sunrise, Fl). Next, the mechanical aortic valve was explanted and surrounding aneurysmal tissue was resected. Given severe aortic valve patient-prosthesis mismatch, replacement with a larger valve was mandatory. However, the aortic annulus was severely calcified, due to the previous Konno procedure, making a root enlargement impossible. Therefore, instead of standard Bentall (aortic root replacement), a skirted Bentall operation was performed by moving the aortic annulus about $8 \mathrm{~mm}$ into the aorta. Thereby, a $23 \mathrm{~mm}$ St Jude's mechanical Regent valve (Copyright Abbott, Chicago, IL) was able to be utilized in a $26 \mathrm{~mm}$ Valsalva graft (copyright Terunmo, Sunrise, FL). The aortic root calcifications also involved the left and right main coronary arteries, making coronary mobilization hazardous. Therefore, the Cabrol procedure was performed, suturing an $8 \mathrm{~mm}$ graft to the left and right main coronary orifice, with a side-to-side anastomosis to the Valsalva graft.

Finally, a CryoMaze probe (copyright Atricure, Mason, $\mathrm{OH}$ ) was used to perform a right sided MAZE for his persistent atrial flutter. This was done by performing a superior to inferior vena cava lesion, connected to a lesion to the right atrial appendage and to the annulus of the tricuspid valve using 
cryothermia. A decision was made not to replace the mechanical mitral valve as the patient lacked symptoms of shortness of breath prior to the $A D$ and the already prolonged and complex operation he needed. The patient did well post-operatively in normal sinus rhythm with no major complications.

\section{Discussion}

Shone's complex is a relatively rare form of CHD, making up about $0.6 \%$ of total CHD cases (6). For patients who are diagnosed during childhood, management involves early surgical repair, with 63\% of patients requiring multiple interventions (8). The variability of lesions in incomplete SC makes the formulation of a generalized surgical strategy problematic. There have been 2 retrospective studies analyzing surgical management of SC to this date which recommend left ventricular outflow tract (LVOT) obstruction to be addressed with initial surgery and that repair of valves should be done whenever possible $(6,8,9)$.

Due to the rarity of this disease presentation, there is limited published literature on SC in adult patients. There is concern that SC may be under-recognized in the adult population due to decreased awareness of the syndrome. There have been multiple case series and reports of patients who were initially diagnosed with isolated CoA or other LVOT obstructions who later presented with progressive mitral valvular disease in adulthood and were found to have undiagnosed SC $(4,10,11)$. A retrospective study by Aslam et al, found that only $39 \%$ of SC cases were diagnosed in childhood and that the mean age of diagnosis was 32 years old (3).

With the improvement of CHD surgical techniques, patients with CHD and specifically SC are living much longer into adulthood. Mortality is generally limited to infancy and case series have shown favorable outcomes with nearly 24 years without clinical events and that up to $90 \%$ of patients are transplant free by 30 years old $(3,10)$. However, due to their complex anatomy and multitude of cardiac surgeries, SC patients have a significantly higher health services utilization compared to adults in the general population. Nearly $50 \%$ of SC patients are hospitalized as adults, most commonly for arrythmias, heart failure, and new surgical interventions (3). This increased morbidity in adulthood may be associated with significant hemodynamic changes from left sided obstructive lesions as studies have shown that SC patients have significant LV remodeling and diastolic dysfunction in adulthood. (12).

Our patient in this case presented with acute aortic dissection in the setting of a slow growing proximal aortic aneurysm treated with a skirted Bentall procedure and aortic valve replacement. To our knowledge, there has only been one other case report of a patient with incomplete SC presenting with a type A dissection years after a CoA repair (11). In our case, the patient should have been referred for 
surgical consult and prophylactic surgery for aortic dilation once the aorta surpassed 5 centimeters. SC patients with AVR during their early life are at high risk for complications. While there are no guideline recommendations for prophylactic surgery specifically for aortopathy-associated CHD in adults, the current STS, AATS, and ACC/AHA guidelines all recommend prophylactic replacement of the aorta once it reaches $5.5 \mathrm{~cm}(13)$.

One major complication for CHD patients in adulthood is patient prosthesis mismatch (PPM), which possibly contributed to further dilation of the aortic aneurysm in this patient. PPM occurs when the area of a functioning prosthetic valve is too small for the body surface area, due to normal somatic growth of cardiac tissue (14). In our case, the patient had both aortic and mitral valve replaced in childhood, which both became too small for his size. It has been shown that severe PPM is associated with a 2-fold increase in mortality as well as worsening functional capacity and higher rates of HF hospitalizations in CHD patients (14). Nearly one third of CHD patients require interventions in adulthood mainly for degeneration of bioprosthetic heart valves and patient-prosthesis mismatch result from somatic growth (3). It is important for physicians to consider these potential complications in SC patients and monitor appropriately, particularly since they are living so much later into adulthood now.

Finally, atrial flutter in patients with CHD can have debilitating symptoms and substantially increases the risk of thrombus formation, stroke, and tachycardia-induced cardiomyopathy. Right-sided MAZE procedure addresses right sided atrial flutter and provides curative treatment of atrial flutter. In patients with concomitant atrial fibrillation, the atrial fibrillation-free success of bi-atrial MAZE procedure is above $80 \%$ at five year follow (15).

\section{Conclusion}

Shone's complex is a rare and underrecognized syndrome associated with relatively low mortality in adulthood but carries substantial morbidities. Given the increasing number of patients with SC surviving to adulthood, it is important to gain a better understanding of the prognosis and tailor appropriate management. This case should bring increased awareness to SC and its potential complications such as atrial flutter and aortic dissection. Further research is needed to determine appropriate guidelines for when to intervene on aortopathy-associated CHD and reliable markers for dissection-risk in CHD patients.

\section{Abbreviations}

SC- Shone's complex 
CHD- Congenital heart disease

CoA- Coarctation of the aorta

PMV- Parachute mitral valve

MV- Mitral valve

LVIT- Left ventricular inflow tract

LV- Left ventricle

AD- Aortic Dissection

ASD- atrial septal defect

VSD- ventricular septal defect

$\mathrm{PH}$ - Pulmonary hypertension

LVOT- left ventricular outflow tract

TTE- transthoracic echocardiography

AVR- aortic valve replacement

PPM- patient prosthesis mismatch

BSA- body surface area

\section{Declarations}

Ethics Approval and Consent to Participate

Not Applicable

Consent for Publication

Informed consent for publication was obtained

\section{Availability of Data and Materials}

Data sharing is not applicable to this case report as no datasets were generated or analyzed in our article.

\section{Competing Interests}

The authors declare that they have no competing interests to report.

\section{Funding}

The authors declare that there is no funding to report.

\section{Authors' Contributions}

SS, SR, SW, FM, and AK were all involved in the management of the patient and participated in the writing of the paper. AK was involved in the operative management of the patient and in the writing of the paper. SR participated in the performance of the research and substantive revise of the work. All authors read and approved the final manuscript and have agreed both to be personally accountable for the author's own contributions and to ensure that questions related to the accuracy or integrity of any part of the work, even ones in which the author was not personally involved, are appropriately investigated, resolved, and the resolution documented in the literature.

\section{Acknowledgements}

Not Applicable 


\section{References}

1. Shone J, Sellers R, Anderson R, Adams P, Lillehei C, Edwards J. The developmental complex of "parachute mitral valve", supravalvular ring of left atrium, subaortic stenosis, and coarctation of aorta. Am J Cardiol. 1963; 11: 714-725

2. Nicholson G, Kelleman M, Uz C, Pignatelli R, Ayres N, Petit C. Late outcomes in children with shone's complex: a single-centre, 20-year experience. Cardiol Young. 2017; 27: 697-705

3. Aslam S, Khairy P, Shohoudi A et al. Shone complex: an under-recognized congenital heart disease with substantial morbidity in adulthood. Can J Cardiol. 2017; 33: 253-259

4. Zhang $X, M a G$, Zheng $L$ et al. Echocardiographic diagnosis of shone's syndrome. Echocardiography. 2020; 37: 2139-2143

5. Jin S, Homsy J, Zaidi S. Contribution of rare inherited and de novo variants in 2,871 congenital heart disease probands. Nature. 2017; 49(11): 1593-1601

6. True A, Baidya M, Lui C, Kilimnik G, Sadler M. Computed tomography imaging characteristics of shone syndrome. Radiol Case Rep. 2019; 14: 164-167

7. Ionescu-Ittu R, Mackie A, Abrahamowicz $M$ et al. Valvular operations in patients with congenital heart disease: increasing rates from 1988 to 2005. Ann Thorac Surg. 2010; 90: 1563-1569

8. St. Louis J, Bannan M, Lutin W, Wile H. Surgical strategies and outcomes in patients with shone complex: a retrospective review. Ann Thorac Surg . 2007; 84: 1357-1363

9. Malhotra $S$, Lacour-Gayet $F$, Campbell $D$ et al. Outcomes of reparative and transplantation strategies for multilevel heart obstructions with mitral stenosis. Ann Thorac Surg. 2008; 86(4): 1305-1310

10. Grimaldi A, Vermi A, Ho S. Surgical outcome of partial shone complex. Interact Cardiovasc Thorac Surg. 2012; 14: 440-444

11. Ahmed M, Aziz H, Jiang L. Severe aortic complications in a patient with variant shone's complex and bicuspid aortic valve. BMJ Case Rep. 2017

12. Jain C, Warnes C, Egbe A. Hemodynamics in adults with the shone complex. AM J Cardiol. 2020; 130: 137-142

13. Hiratzka L, Bakris G, Beckman J, et al. 2010 ACCF/AHA/AATS/ACR/ASA/SCA/SIR/STS/SVM guidelines for the diagnosis and management of patients with thoracic aortic disease. Circulation. 2010; 121; 266-369

14. Pibarot P, Magne J, Leipsic J. Imaging for predicting and assessing prosthesis-patient mismatch after aortic valve replacement. JACC Cardiovasc Imaging. 2019; 12(1): 149-162

15. Ad N, Holmes S, Friehling T. Minimally invasive stand-alone cox maze procedure for persistent and long standing persistent atrial fibrillation perioperative safety and 5 year outcome. Circ Arrhythm Electrophysiol. 2017; 10 (11). 


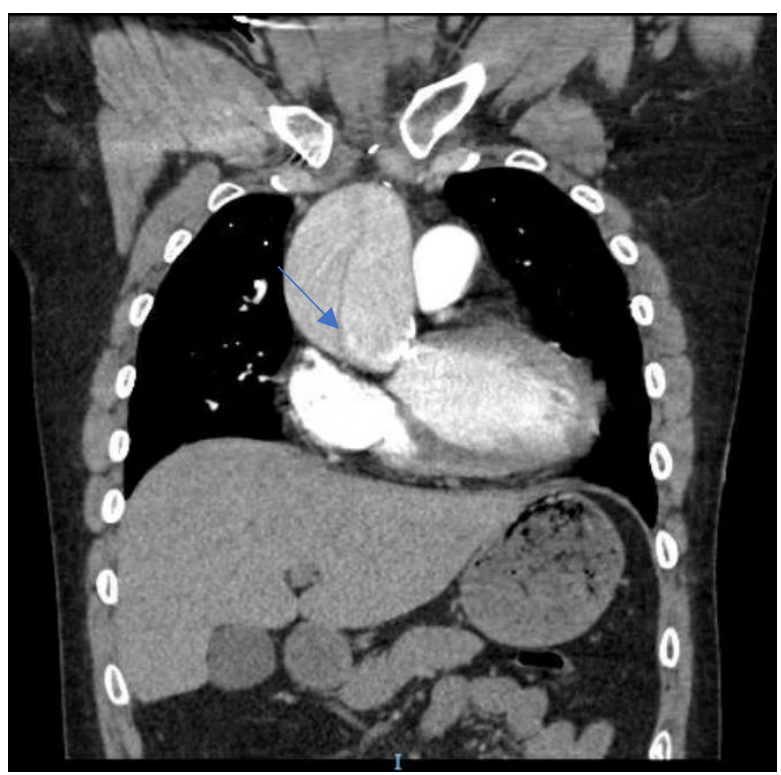

Figure 1 CTA chest coronal view at OSH demonstrating acute aortic dissection in setting of chronic aortic dilation (blue arrow)

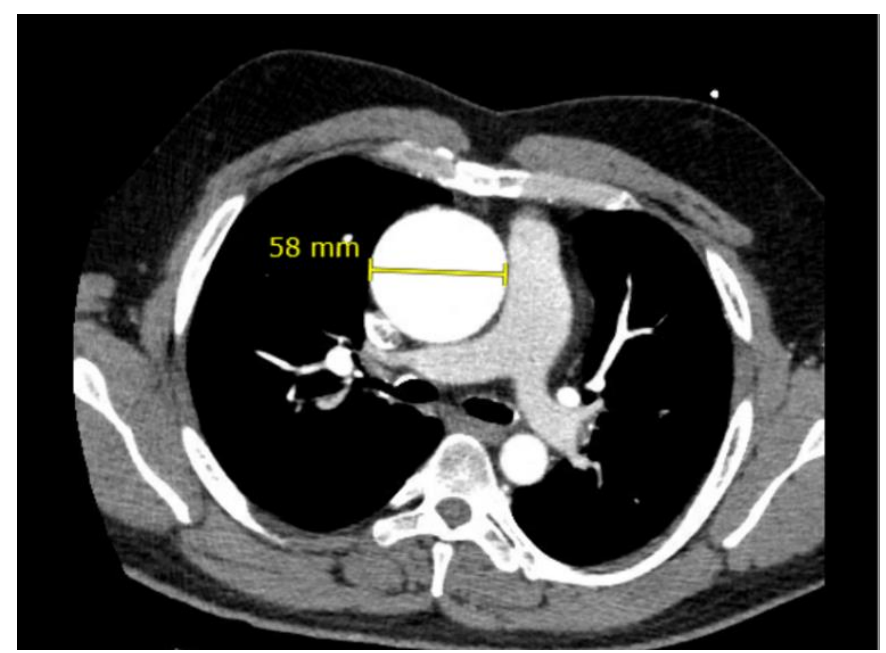

Figure 2 CTA chest axial cut demonstrating stable fusiform aneurysmal dilation of ascending thoracic aorta measuring $5.8 \mathrm{~cm}$ in maximum transverse diameter, mildly increased from previous $C T$ 4 mo prior. No evidence of aortic dissection or hematoma

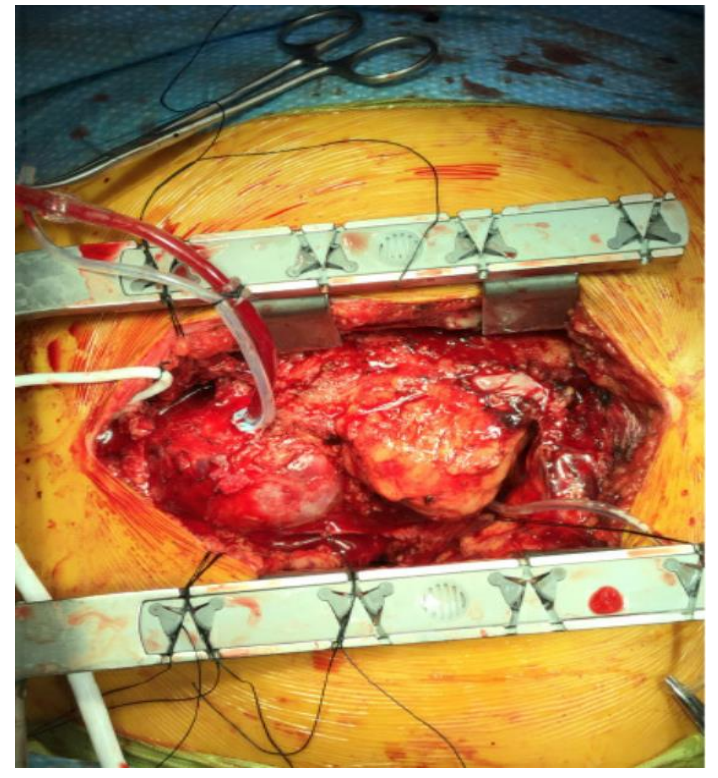

Figure 3 OR view of re-do sternotomy with large aortic root aneurysm and acute type $A$ aortic dissection 


\section{Supplementary Files}

This is a list of supplementary files associated with this preprint. Click to download.

- CAREchecklistEnglish2013.pdf 